\title{
Reforestación y conservación de árboles nativos en el corregimiento de San Rafael municipio de San Sebastián, Magdalena ${ }^{1}$
}

\section{Reforestation and conservation of native trees in the Municipality of San Rafael Municipality of San Sebastián Magdalena}

DOI: 10.17981/mod.arq.cuc.20.1.2018.09

Artículo. Fecha de recepción: 05/06/2017 Fecha de aceptación: 29/10/2018

Ambrosio García²,

Claudia Echeverría, Edgardo Montero, Elena Villar, Ismael Arévalo, Jaider Hernández, Viviana González, José Rubio, Rodrigo Carvajal y Luis Baños

IED Luis Millán Vargas (Colombia)

cristanlagreso@gmail.com

Para citar este artículo:

García, A., Echeverría, C., Montero, E., Villar, E., Arévalo, I., Hernández, J., González, V., Rubio, J., Carvajal, R. y Baños, L. (2018). Reforestación y conservación de árboles nativos en el corregimiento de San Rafael municipio de San Sebastián, Magdalenan. MODULO ARQUITECTURA-CUC, vol. 20, no. 1, pp. 95-108. DOI: 10.17981/mod.arq.cuc.20.1.2018.09

\section{Resumen}

El objetivo de esta investigación fue dar a conocer el estado actual de la deforestación, como una de las acciones de hombre en el uso indiscriminado de los recursos de la naturaleza, especialmente en la tala y quema de bosques. Lo que ha afectado el medio ambiente, generando la desaparición paulatina de muchas especies importantes de la flora y la fauna, entre otras. El presente estudio se realiza desde una mirada cualitativa, describiendo las causas que han obligado a la comunidad de San Rafael a destruir con diferentes actividades los árboles nativos de importantes especies en el entorno. Para el logro de los objetivos se aplicaron entrevistas estructuradas a los estudiantes y pobladores del municipio. Como conclusiones es importante mencionar que la deforestación en el municipio de San Sebastián ha disminuido en comparación con años anteriores, sin embargo, se debe seguir trabajando en temas de educación ambiental.

Palabras claves: Deforestación; recursos naturales; degradación; sequedad; flora y fauna; árboles nativos.

\section{Abstract}

The objective of this research was to present the current state of deforestation, as one of the actions of man in the indiscriminate use of natural resources, especially in the felling and burning of forests. What has affected the environment, generating the gradual disappearance of many important species of flora and fauna, among others. The present study is carried out from a qualitative perspective, writing the causes that have forced the community of San Rafael to destroy with different activities the native trees of important species in the environment. In order to achieve the objectives, a structured interview was applied to the students and residents of San Rafael. As conclusions, it is important to mention that deforestation in the municipality of San Sebastián has decreased compared to previous years, however, work should continue on environmental education issues.

Keywords: Deforestation; natural resources; degradation; dryness; Flora and fauna; Native trees

${ }^{1}$ Este artículo ha sido derivado del Programa de Fortalecimiento de la Cultura Ciudadana y Democrática CT+I a través de la IEP apoyada en TIC en el Departamento de Magdalena: CICLÓN. Desarrollado con docentes miembros del grupo de investigación "Defensores de los Bosques" pertenecientes a la Institución Educativa Departamental Luis Millán Vargas.

${ }^{2}$ Líder del grupo de investigación "Defensores de los Bosques". 
La deforestación es un tema de gran importancia a través de la historia, siendolo todavía medioambiental que se está viviendo en el planeta, se requiere de una mayor atención con todo lo que este relacionado con el entorno, razón por la cual el objetivo de esta investigación es dar a conocer el estado actual de la deforestación, como una de las acciones del hombre en el uso indiscriminado de los recursos de la naturaleza, especialmente la tala y quema de los bosques. Lo que ha afectado el medio ambiente, suscitando la degradación fértil del suelo, la sequedad de fuentes hídricas, contribuyendo a la alteración del clima, la desaparición paulatina de muchas especies importantes de la flora y la fauna, entre otras
razones.

Considerando la necesidad de reforestación, conservación y recuperación de árboles nativos, este proyecto se enfoca en han obligado a la comunidad de San Rafael a destruir con diferentes actividades a los árboles nativos de importantes especies en el entorno.

Hoy en día, los bosques y selvas de todo el mundo conforman una parte significativa de la superficie terrestre; no obstante, cada año millones de hectáreas son deforestadas

Fig. 1. Fotografia de la tala de un and
e: htps://s3./smazonaws.com/files. pucp.

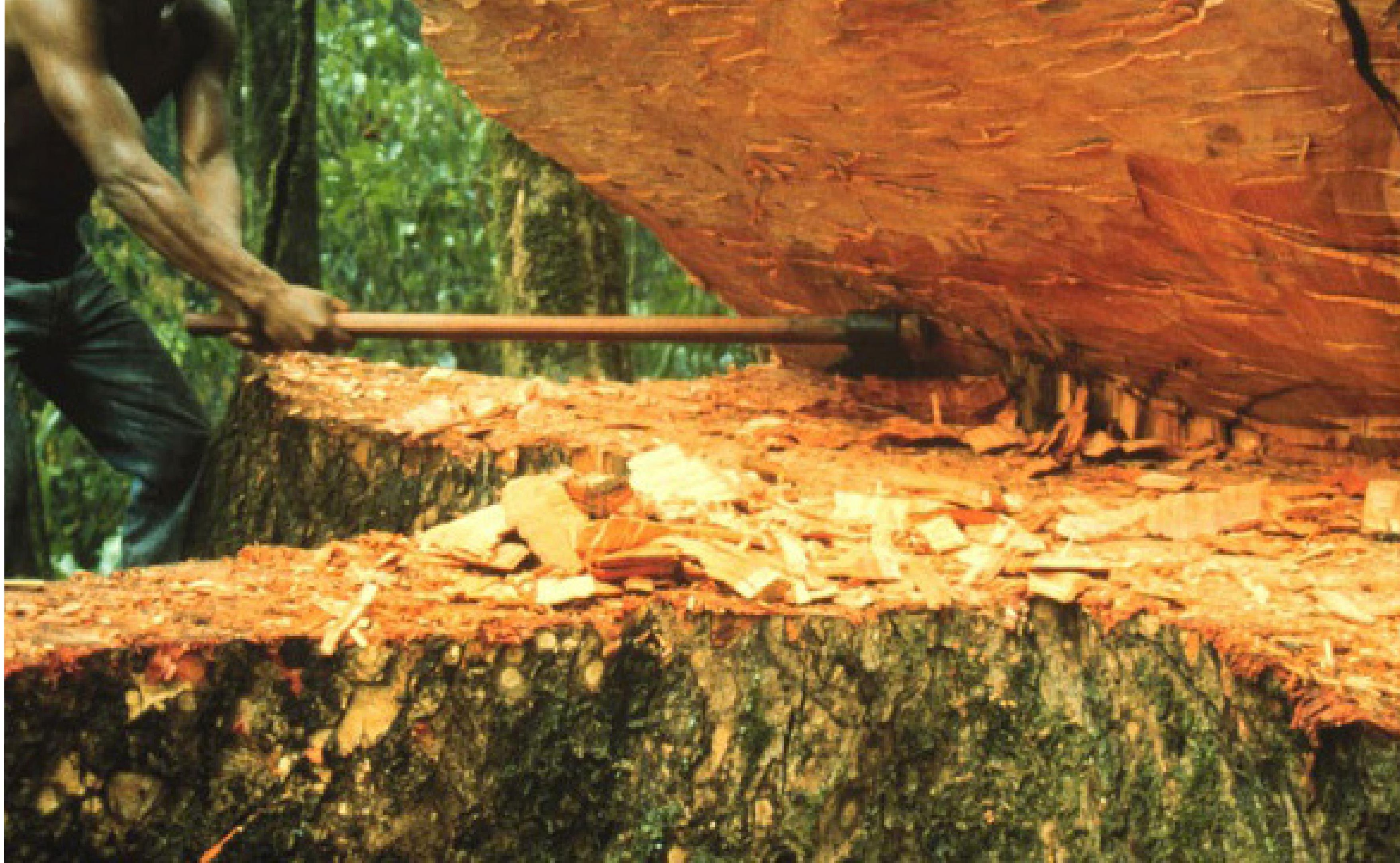


por consecuencia directa de las actividades humanas, lo cual trasciende de la escala local, regional hasta una escala mundial. La tala de bosques siempre ha sido un factor negativo, pues la tasa de deforestación, evidencia lo antes planteado. (Renovables verdes, 2017). Razón por la cual es necesario la conservación de los árboles en espacios urbanos y rurales, al ser estos los encargados de limpiar el aire a través de la fotosíntesis, capturando el gas carbónico $\left(\mathrm{CO}_{2}\right)$ para integrarlo a su biomasa: tallos, ramas, hojas y raíces. Además, utilizando los árboles como medios naturales de acondicionamiento microclimático, numerosos beneficios aparecen: sombra tan necesaria en nuestro clima, canalización de los vientos y enfriamiento pasivo de los ambientes (Rodríguez, 2011).

En el año se pierden 13 millones de bosques en el mundo, específicamente, en Asia, África y América. En Colombia el Instituto de Hidrología, Meteorología y Estudios Ambientales-IDEAM informó que el país perdió 178.597 hectáreas de bosques, es decir un aumento del 44\% frente al 2015 (El Espectador, 2017). Siendo los departamentos más afectados: Caquetá, Chocó, Meta, Antioquia, Norte de Santander, Guaviare y Putumayo; zonas donde afloran los cultivos ilícitos, el tráfico ilegal de madera, la ganadería y la expansión de la frontera agrícola, factores que han conllevado al aumento significativo en el descalabro forestal del país.

El departamento del Magdalena se encuentra entre los diez (10) departamentos con menor deforestación del país, con una tasa que solamente alcanza las 1.378 hectá- reas de bosque (República de Colombia. Ministerio del Medio Ambiente y Desarrollo Sostenible-Minambiente, 2017).

El corregimiento de San Rafael, jurisdicción del municipio de San Sebastián cuenta con una vasta región netamente agropecuaria en la que los colonos y terratenientes con aras de sostener e incrementar las especies bovinas, equinas, y porcinas, entre otras, han empleado técnicas inapropiadas en el aprovechamiento de los suelos incurriendo en la destrucción del ecosistema, por la tala, quema y aplicación de herbicidas en potreros que han causado la disminución significativa de los árboles nativos; y generando como consecuencia la infertilidad de la capa vegetal del suelo, escasez de lluvia, sequías de fuentes hídricas, calentamiento, contaminación ambiental y la poca disponibilidad de terrenos aptos para la agricultura tradicional, que sirve para el sustento diario de las familias de la región.

\section{Reforestación}

Hace poco más de medio siglo, surge el concepto de reforestación, como una salida a la paulatina destrucción del medio ambiente y al cambio climático existente. $Y$ se define como la actividad de plantar árboles donde una vez existía bosque, con el objetivo primordial de incrementar el número de especies, para conservar el medio ambiente, preservar las zonas verdes y por consiguiente aumentar las especies nativas de flora y fauna, considerando tener un espacio limpio que beneficie a la comunidad (Vargas, 2012). 
Los árboles son parte fundamental de los ecosistemas, por el sinnúmero de beneficios ecológicos que estos generan. Según Reyes y Gutiérrez (2010).

“Los árboles protegen de los rayos solares, tamizan la luz intensa y embellecen el espacio, pero, sobre todo, tienen una función de regulación bioclimática que favorece la humedad del aire al absorber el agua por sus raíces y restituirla por evapotranspiración" (p. 24).

Lizana citada por Reyes y Gutiérrez (2010) afirma que dentro de las ventajas y beneficios que dan los árboles se encuentra la radiación solar, siendo opacada con la sobra, lograr absorber calor, y generarcontrol de la temperatura. Por otro lado, según Vining y Ebreo (1992) aseguran que es necesario cuidar y proteger los árboles, puesto que son estos los que brindan bienestar físico y psíquico a los individuos. Por tal razón, los seres humanos deben aprender a plantar de manera adecuada los árboles, así como también garantizarles la protección, para el futuro suministro de sombra y oxígeno.

Según la tipología de árboles que se desean plantar, resulta fundamental resaltar que el aporte de nutrientes tendrá en cuenta las características de las especies, según el tipo; por ejemplo las leguminosas, caducifolias, contenidas de nutrientes en sus hojas, sistema radicular, degradación de la hojarasca, etc. Los entes encargados deben contar con la experiencia requerida para relacionar variables e integrar variables ambientales y técnicas (Chávez, s.f.).

Con el paso de los años se ha notado fuertemente un accionar despreocupado, egoísta y contaminante por parte del hombre sobre los recursos; sin embargo, actualmente se ha hecho consciente que los daños causados afectan directamente el accionar y las relaciones humanas. Es por esto que se puede inferir que los pensamientos sociales, culturales y económicos han permitido trascender de conductas que anteriormente dominaban el comportamiento de los individuos.

Fig. 3. Argentina: Guardianes de árboles autóctonos.

Fuente: http://agriculturers.com/wp-content/uploads/2015/10/algarrobo.jpg






\section{Conciencia y educación Ambiental sostenible}

Es importante generar conciencia de la conservación de los recursos naturales, desde la transversalidad de la escuela como ente de formación, inculcando desde la formación de los más pequeños la generación de conciencia sobre la conservación de los recursos naturales; que se pueden utilizar, pero con la implementación de estrategias para que el uso sea sostenible y no se siga deteriorando a la naturaleza, y con ello, las generaciones futuras también puedan disfrutar de unos ecosistemas sanos. Considerando que los estudiantes se convierten en replicadores de los conceptos adquiridos, siendo promotores ambientales en la reforestación y conservación de las especies vegetales nativas, inicialmente, y así se crea una cultura de conservación de los demás recursos naturales por todos y cada uno de los miembros de la comunidad (Sauvé, 1999).

\section{Metodología}

La investigación estuvo orientada por el paradigma crítico-social, al fundamentarse en la reflexión crítica, buscando reformar a las instituciones que hacen parte de la realidad social a la que pertenecen los investigadores, La investigación fue de tipo cualitativo, y como lo señala Cerda (2007), tuvo como objetivo la descripción de las cualidades de estos fenómenos.

\section{Técnicas e instrumentos de recolección de la información}

Se emplearon como técnicas e instrumentos de recolección de la información a las entrevistas estructuradas, que constaban de diez (10) preguntas abiertas con base a las categorías de estudio, así mismo se emplearon la observación participante, y además se elaboró un diario de campo con las actividades realizadas a lo lago del proyecto.

\section{Escenarios y actores del estudio}

El escenario en el que se desarrolló la investigación es la IED Luis Millán Vargas del municipio de San Sebastián-Magdalena, con la participación en el estudio de (40) sujetos (20) estudiantes de diferentes grados y (20) miembros de la comunidad.

\section{Procedimiento}

Momento (1): Se seleccionan los instrumentos a utilizar.

Momento (2): Se especifican las categorías de estudio.

Momento (3): Se escogen los instrumentos de recolección de información.

Momento (4): Con base a las categorías de estudio: Reforestación, árboles nativos, y estrategias educativas pedagógicas se aplicaron entrevistas, las cuales describen el fenómeno que se estudia partiendo del nivel de la investigación (no experimental) en donde se observa y analiza, tal y como acontece. 
Momento (5): Una vez se tiene los resultados de la entrevista estructurada y la observación registrada se interpretan y comprenden los resultados a la luz de lo que exponen los teóricos que soportan la investigación.

Momento (6): Se relacionan los resultados y se muestran los hallazgos.

\section{Resultados}

A continuación, se presentan lo hallazgos derivados del instrumento aplicado (Tabla 1).

Para estos hallazgos se tuvo en cuenta la pregunta orientadora: ¿Qué estrategias se pueden implementar para contrarrestar la disminución progresiva de árboles nativos en la comunidad de San Rafael?

Tabla 1

Resultados entrevistas ¿Qué estrategias se pueden implementar para contrarrestar la disminución progresiva de árboles nativos en la comunidad de San Rafael?

\begin{tabular}{|c|c|c|c|}
\hline $\begin{array}{l}\text { Categoría } \\
\text { de estudio }\end{array}$ & $\begin{array}{c}\text { Pregunta } \\
\text { orientadora }\end{array}$ & Discurso de los actores & Articulación y sistematización teórica \\
\hline Reforestación & $\begin{array}{l}\text { ¿Cuáles son las } \\
\text { situaciones que } \\
\text { se presentan } \\
\text { por cuenta de la } \\
\text { deforestación? }\end{array}$ & $\begin{array}{l}\text { "Se han disminuido las clases de } \\
\text { árboles" "Tala y quema de árboles } \\
\text { de montaña" "La gente tala los } \\
\text { árboles y no siembra para recuperar } \\
\text { la forestación y que los animales } \\
\text { puedan vivir los animales" "Tala } \\
\text { de árboles para fabricar muebles" } \\
\text { "Escasez de agua, ya que dificulta } \\
\text { la supervivencia de especies } \\
\text { nativas en el corregimiento" } \\
\text { "Desaparición de la fauna, } \\
\text { aumento de la temperatura, aire } \\
\text { contaminado porque los árboles } \\
\text { no lo purifican" "Calentamiento, } \\
\text { desaparición de flora y fauna, } \\
\text { pocas precipitaciones" "Por la tala } \\
\text { se pierde la sombra, se contamina } \\
\text { más el oxígeno" "Escasez de agua } \\
\text { en los pozos y caños" "Tala para } \\
\text { leña y cocinar" "Malos olores" } \\
\text { "Que sean resistentes al clima y de } \\
\text { rápida reproducción" "Expuestos } \\
\text { a enfermedades" "Falta de fuentes } \\
\text { hídricas" "Afectación del suelo y } \\
\text { extinción de animales" "Erosión } \\
\text { de suelos" "Infertilidad de suelos, } \\
\text { pérdida de árboles nativos." }\end{array}$ & $\begin{array}{l}\text { Navarro en el (2016) afirma que existe un crecimiento } \\
\text { desorganizado de las sociedades, que en gran medida } \\
\text { ha traído repercusiones significativas, y es el tema medio } \\
\text { ambiental quien no se aleja de estos resultados de impacto; } \\
\text { por lo anterior y de acuerdo a los hallazgos encontrados } \\
\text { en la entrevista aplicada, se deduce que los estudiantes } \\
\text { se quejan de la deforestación porque hay un deterioro del } \\
\text { medio ambiente, afectando el cambio climático en cuanto a } \\
\text { flora, fauna, fuentes hídricas, especies nativas y aumentando } \\
\text { el calor, los malos olores, el aire contaminado, entre otros, } \\
\text { alterando a tal punto el ecosistema que prácticamente no hay } \\
\text { un hábitat en el cual los animales puedan vivir. } \\
\text { Frente a esto, autores como Rodríguez (2011) consideran la } \\
\text { reforestación como la actividad de plantar árboles donde una } \\
\text { vez existía bosque, su objetivo primordial es el de incrementar } \\
\text { el número de especies para conservar el medio ambiente, } \\
\text { preservar las zonas verdes y por consiguiente aumentar las } \\
\text { especies nativas de flora y fauna, considerando tener un } \\
\text { espacio limpio que beneficie a la comunidad. } \\
\text { Lo que quiere decir Rodríguez (2011) es que, si se realiza } \\
\text { la actividad de reforestación, todas estas inconformidades } \\
\text { o quejas que tienen los estudiantes sobre el daño que se le } \\
\text { está haciendo al planeta al acabar con los ecosistemas, en } \\
\text { este caso con la tala de árboles nativos, puede cambiar y el } \\
\text { mismo se puede salvar, brindando una mejor calidad de vida } \\
\text { no solo para los animales sino también para los humanos. }\end{array}$ \\
\hline
\end{tabular}


Árboles

nativos
¿Qué estrategias de difusión aplicaría para sembrar y conservar árboles en el corregimiento de San Rafael?

"Hablar con la comunidad para convencerlos de sembrar árboles" "Prohibir la tala y sembrar árboles" "Sembrar árboles, regarlos y hacer mejor la vida para los animales" "Haciendo charlas con los padres de familia que colaboren conservando árboles" "Colocar afiches con mensajes de conservación de árboles, los estudiantes hacer charlas con los dueños de las fincas para concientizarlos del daño que hacen" "Colocar pancartas acerca de como conservar árboles, en la radio anunciar los cuidados que se deben tener" "Campañas" "Hablar con el alcalde para que done un lote para reforestar" "Apoyarse en las TIC para difundir" "Sembrar 5 árboles en cada casa y por cada árbol talado sembrar 3" "Sembrar en sitios adecuados"

"No talar árboles", "Marcando las clases de árboles que dan utilidad" "Haciendo una

¿Cómo se

pueden seleccionar las especies de árboles para llevar a cabo la reforestación en el corregimiento de San Rafael? investigación de árboles que han ido desapareciendo" "Averiguar las especies nativas que han disminuido desde hace 30 años" "Buscando especies nativas en otros territorios" "árboles que den frutos" "La más rápidas en crecimiento, las que hagan más sombra, que sea nativo y de buena madera" "Los que se mantengan vivos en verano".

A la luz de lo expresado por los entrevistados se evidencia que para ellos la reforestación empieza con la convicción de que para mejorar el ecosistema se deben plantar árboles, por lo que la mayoría concuerda en sembrar árboles en su casa y en la comunidad, mientras que al mismo tiempo, se hacen charlas y campañas sobre la importancia del mismo, utilizando no solo pancartas y medios de comunicación sino también las TIC para difundir el mensaje.

Las respuestas brindadas a través de la entrevista contribuyen a lo que se afirma en el proyecto "Recuperar especies de plantas nativas, con la siembra y cuidado de estas, en los espacios verdes de la comunidad de Tunibamba, Parroquia el Sagrario, Cantón Cotacachi, de la provincia de Imbabura". En el que se sostiene que el problema está en escasa sensibilización que reciben los miembros de la comunidad en lo relacionado con conservación y cuidado que deben de tener con el cuido las plantas nativas.

Lo anterior deja ver que existe claridad sobre los planteamientos de los estudiantes en la entrevista, puesto que concuerdan en que se debe sensibilizar a la comunidad por el medio que sea sobre la conservación de las plantas nativas para poder cuidar los ecosistemas donde viven los animales.

Las respuestas expuestas por los estudiantes hacen notorio que es necesario investigar todo tipo de árboles nativos o no, que puedan crecer en la región para salvaguardar el ecosistema y que no solo den sombra y sirvan para realizar objetos en madera, sino también ofrezcan algún tipo de fruta que sirva de alimento de las distintas especies que puedan ser atraídas por ellas.

En el proyecto "Recuperar especies de plantas nativas, con la siembra y cuidado de estas, en los espacios verdes de la comunidad de Tunibamba, Parroquia el Sagrario, Cantón Cotacachi, de la provincia de Imbabura", reafirma las conclusiones explícitas por los estudiantes cuando expone que el desconocimiento por parte de las personas sobre el cuidado y la utilidad que se le deben dar a las plantas, específicamente las nativas; situación que ha que ha ocasionado extinción de las plantas, provocando significativos problemas medioambientales. Lo anterior quiere decir que ahora la gente aprecia el valor de los árboles por lo que sensibilizarlos es más sencillo y estarán más interesados en investigar que tipo de plantas pueden ser cultivadas en la región y por ende cultivarlas, y así todos los daños ambientales que se han causado hasta el día de hoy puedan verse disminuidos. 
Cont..

¿Cómo

se puede garantizar

Reforestación una eficaz reforestación, protección y conservación?

¿Por qué cree usted que algunas

Árboles nativos
"Prohibiendo todo lo que daña a los árboles" "Abonando, cuidando regando, conservando y ayudarlos a crecer" "Sembrando árboles" "Siembra de árboles abonándolos" "Sembrar toda clase de árboles, hacer control de plagas" "Con guardias de bosques" "Creando grupos para que estén pendiente de lo que sucede con los árboles plantados".

"Porque no los cuidamos y los cortamos" "Porque se ha destruido su hábitat" "Cuando se talan árboles las especies se extinguen" "Porque se han destruido los árboles" "Tala, quema" "Porque algunos dueños de fincas por hacer pastizales para los animales" "Se han disminuido por las industrias madereras, la ganadería y otras especies nativas han disminuido o se han desaparecido? actividades" "Para construcción de casas, corrales, negocios ilícitos" "Crecimiento de la población" "Mal uso" "Por el narcotráfico" "Porque maltratamos los árboles" "Por la quema de las montañas" "Cambios climáticos, escasez de agua por pocos árboles"

"Invasión de territorios, tala y quema de árboles" "Por la acción del hombre" "Para que los animales tengan donde vivir".
Los estudiantes reconocen que para salvar el ecosistema es necesario no solo sembrar árboles sino también proteger los que aún no se han talado, ubicando puntos de vigilancia, no solo de "guardabosques" sino también concientizando a la misma comunidad para que ellos se apropien del proceso y sean ellos mismos quienes siembren y protejan la naturaleza.

Resulta también relevante anotar que los resultados permiten sumar a lo expuesto por García, Ramos y Becerra (2011), en donde afirman que son las fuentes semilleras una solución al rescate y conservación forestal. Se destaca entonces la necesidad de realizar cambios en la comunidad, que conduzcan a la conservación y protección del ecosistema, mediante la investigación y el rescate no solo de las especies nativas sino de nuevas especies que puedan crecer en la zona.

De acuerdo a los hallazgos encontrados en la entrevista aplicada, se deduce que los estudiantes consideran básicamente que todas las actividades lideradas por el hombre ya sea mediante la tala, la quema, o negocios mediante la industria de madera son directamente los responsables de la desaparición de las especies nativas, y de la destrucción de ecosistemas y hábitats de los animales.

Frente a esto el IDEAM (2017) afirma que los bosques naturales, actualmente están distribuidos en las cinco principales regiones biogeográficas del país o regiones naturales, pero se encuentran en mayor proporción en la región de Amazonía (66,9\%) y Andina (17,8\%), seguidos por las regiones Pacífica (8,8\%),Orinoquía (3,6\%) y Caribe (2,9\%). Adicionalmente, los grupos étnicos en Colombia son titulares de territorios donde se encuentra el $53,4 \%$ de los bosques naturales en Colombia, encontrando el $46,1 \%$ en resguardos indígenas y el $7,3 \%$ en territorios colectivos de comunidades negras o afrocolombianas.

Por lo que lo anterior, se confirma que evidentemente la cantidad de árboles nativos de cada región se ha visto disminuida e incluso extinta en gran proporción y prácticamente las pocas zonas verdes que aún quedan en el país son aquellas que están resguardadas por comunidades indígenas o afrocolombianas; comunidades que consideran la naturaleza como pura y primordial. 
A la luz de lo expresado por los estudiantes se puede observar la percepción que tienen sobre la investigación de como el clima en la región se ha modificado gracias a la tala másiva de los árboles, puesto que la temperatura cada vez está en aumento,

"Un clima agradable con pocas clases de árboles" "Cada vez es más caluroso" "Hace mucho calor"

¿Qué cambios ha notado usted en el clima del

Estrategias educativas corregimiento de San Rafael en los últimos 40 años?

"Árboles disminuidos, Temperatura aumentada" "Hace 40 años esta era una zona boscosa con hay escasez de Iluvia" "Aumento de la temperatura" "Ha cambiado el tiempo de verano e invierno" temperatura más baja que la actual, "No he visto cambio" "Ya no se ven telarañitas en las cercas con los rocíos de la noche" "Hay poco invierno y más verano". produciendo más humedad y sensación de calor, por lo que en algún momento no habrá la suficiente vegetación para mantener fresca la zona y el verano será cada vez más largo, secándose la región, llegando incluso a poder convertirse en un desierto.

Las respuestas brindadas a través de las entrevistas contribuyen a lo que afirman en el proyecto "Recuperar especies de plantas nativas, con la siembra y cuidado de estas, en los espacios verdes de la comunidad de Tunibamba, Parroquia el Sagrario, Cantón Cotacachi, de la provincia de Imbabura", en donde se observa que la mayoría de los actores contribuyen significativamente a los procesos de reforestación.

Lo anterior deja ver que existe claridad sobre como la misma comunidad siente y percibe el daño que le hacen al medio ambiente, pues sienten los cambios climáticos en cuanto al hacer más calor pues el sol se siente con más fuerza y cuando hay temporada de lluvias estas son más fuertes, lo que causa desbordamientos en todas las fuentes hídricas, devastando todo a su paso.

Las respuestas expuestas por los estudiantes redundan en la causalidad del ser humano sobre el cambio climático y la desaparición de las especies nativas de la región, puesto que son quienes talan, dañan y no siembran los árboles arrancados de la naturaleza por su mano.

El Espectador (2017) reafirma las conclusiones explícitas por los estudiantes cuando expone que desafortunadamente, los patrimonios nacionales constituidos por los bosques naturales,

"Porque no cuidamos la naturaleza"

¿Por qué cree usted que se den estos cambios climáticos?
"Por la tala y la quema" "Por "Deforestación" "Quema y tala" "Conseguir semillas". la tala y no siembra" "Tala" "Donde hay árboles, llueve" vienen siendo afectado por la deforestación paulatinamente.

Adicionalmente, en el país se aprovechan cerca de 500 especies forestales, para suplir un consumo de madera por año en productos forestales. Se estima que desde el año 1990 hasta el 2016 han ido deforestadas más de 6 millones de hectáreas incidiendo en la pérdida invaluable de biodiversidad y servicios eco-sistémicos, la afectación de espacios vitales para el buen vivir de las comunidades locales, el detrimento de recursos económicos, y el aumento de emisiones de Gases Efecto Invernadero (GEI).

Todo lo anteriormente expuesto refrenda la teoría de como la mano del hombre es la que ha causado toda la tala indiscriminada de las especies nativas para su beneficio, ocasionando una perdida invaluable de la biodiversidad y los ecosistemas. 


\begin{tabular}{|c|c|c|c|}
\hline $\begin{array}{l}\text { Estrategias } \\
\text { educativas }\end{array}$ & $\begin{array}{l}\text { ¿Cree usted } \\
\text { que se pueden } \\
\text { recuperar las } \\
\text { especies nativas } \\
\text { que se han } \\
\text { desaparecido? } \\
\text { ¿Cómo? }\end{array}$ & $\begin{array}{l}\text { "Si, sembrando semillas de árboles } \\
\text { que cortemos" "No se puede } \\
\text { recuperar" "Si" "Sembrando las } \\
\text { especies que se han disminuido, } \\
\text { haciendo un vivero de las especies } \\
\text { que han desaparecido" "Con } \\
\text { semilleros". }\end{array}$ & $\begin{array}{l}\text { Los estudiantes reconocen que las especies nativas se pueden } \\
\text { recuperar en la zona, quizá no recuperar las que ya se talaron, } \\
\text { como lo expresa uno de ellos, sino sembrando árboles de las } \\
\text { mismas especies que se han talado, haciendo viveros y hasta } \\
\text { bosques de dichas especies. } \\
\text { Resulta también relevante anotar que los resultados de las } \\
\text { entrevistas aplicadas a los estudiantes estan de acuerdo con la } \\
\text { información del Instituto Geográfico Agustín Codazzi-IGAC (2012), } \\
\text { que la vocación del suelo en Colombia es en su mayoría forestal } \\
\text { (54\%) seguida por la agrícola (19\%) y la ganadera (13\%). } \\
\text { Se destaca entonces la necesidad de realizar campañas de } \\
\text { reforestación en las que participe toda la comunidad, puesto } \\
\text { que el suelo es lo suficientemente fértil para hacer que crezcan } \\
\text { las especies nativas sembradas, y la comunidad ya reconoce } \\
\text { como las semillas de las especies nativas pueden lograr muchos } \\
\text { cambios con el transcurrir del tiempo y de muchos cuidados. }\end{array}$ \\
\hline $\begin{array}{l}\text { Estrategias } \\
\text { educativas }\end{array}$ & $\begin{array}{l}\text { ¿Por qué } \\
\text { considera } \\
\text { usted que es } \\
\text { importante } \\
\text { implementar } \\
\text { acciones de } \\
\text { reforestación en } \\
\text { el corregimiento } \\
\text { de San Rafael? }\end{array}$ & $\begin{array}{l}\text { "Para que no se pierdan las } \\
\text { especies" "Porque aún existe } \\
\text { vegetación" "Sembrando árboles } \\
\text { tenemos vida" "Para que la } \\
\text { temperatura disminuya y hayan } \\
\text { más especies" "Para volver a tener } \\
\text { un clima como hace } 40 \text { años y } \\
\text { conservar la flora y la fauna" "Para } \\
\text { respirar aire puro". }\end{array}$ & $\begin{array}{l}\text { De acuerdo a los hallazgos, se deduce que los estudiantes } \\
\text { consideran la importancia de hacer campañas de reforestación } \\
\text { para recuperar la fauna y la flora de la región, además de traer } \\
\text { nuevas especies, un clima más agradable y un aire más puro. } \\
\text { Frente a estas razones el IDEAM (2017) afirma que los } \\
\text { ecosistemas boscosos naturales en Colombia, son una de las } \\
\text { condiciones por las cuales el país es catalogado como el segundo } \\
\text { con mayor biodiversidad del mundo, al contar con una extensa } \\
\text { cobertura en bosques naturales que representan el } 52 \% \text { del área } \\
\text { total de la superficie continental, que lo convierte en el tercero } \\
\text { suraméricano, con mayor área en bosques naturales, y con } \\
\text { potenciales condiciones para incentivar su desarrollo forestal. } \\
\text { Lo anterior permite a la comunidad tener la convicción de que si } \\
\text { se realizan campañas de reforestación en la comunidad, esta va } \\
\text { a progresar puesto que el suelo es lo suficientemente fértil como } \\
\text { para que la creación de los bosques nativos salga adelante. }\end{array}$ \\
\hline $\begin{array}{l}\text { Estrategias } \\
\text { educativas }\end{array}$ & $\begin{array}{l}\text { ¿De qué } \\
\text { manera usted } \\
\text { participaría en } \\
\text { el proyecto de } \\
\text { reforestación? }\end{array}$ & $\begin{array}{l}\text { "Apoyando el proyecto" "Cuidando } \\
\text { los árboles y los animales" } \\
\text { "Sembrando árboles", "Creando } \\
\text { un grupo en el corregimiento } \\
\text { para sembrar árboles" "Viveros" } \\
\text { "Dando ideas comprando } \\
\text { árboles" "Donando tierras para la } \\
\text { reforestación". }\end{array}$ & $\begin{array}{l}\text { Datos recientes del Instituto de Investigación de Recursos } \\
\text { Biológicos Alexander von Humboldt (IAVH, 2003) considera la } \\
\text { importancia de los bosques para el desarrollo nacional; por lo } \\
\text { que el Gobierno de Colombia, ha venido promoviendo una serie } \\
\text { de iniciativas dirigidas a impulsar la conservación, uso y manejo } \\
\text { sostenible de estos ecosistemas y controlar la deforestación. } \\
\text { Las respuestas brindadas contribuyen al planteamiento de } \\
\text { proyectos de reforestación en Colombia, convirtiéndola en un } \\
\text { ejemplo a citar en la comunidad, funcionando como incentivo } \\
\text { para concientizar la participación en campañas dentro de la } \\
\text { comunidad, con aportes de los entes territoriales de la región. }\end{array}$ \\
\hline
\end{tabular}




\section{Conclusiones}

Los estudiantes entrevistados reconocieron la importancia de la reforestación y propusieron ideas, estrategias y propuestas para participar de este tipo de proyectos, más aún cuando ellos mismos perciben las alteraciones del cambio climático y caen en cuenta que los seres humanos son los causantes de dicha devastación a la biodiversidad y son los únicos que pueden reestablecerlas.

En la comunidad de San SebastiánMagdalena, la deforestación como causa de impacto medioambiental ha disminuido, lo que a su vez ha logrado que la comunidad en los últimos años tome conciencia en abandonar la tala de árboles, debido a la fallas en la conservación de los entornos verdes y sostenibles.

La mayoría de la comunidad aprecia como ha disminuido notablemente la deforestación en los últimos años; siendo uno de los factores que puede ayudar a entender la disminución en la cantidad de bosques nativos que se pueden talar, ayudando a la toma de conciencia por parte de la población al no cortar los árboles.

Finalmente, este proyecto busca impactar positivamente y de manera integral al municipio de San Sebastián (Magdalena) y trascender el alcance al plano regional, nacional e internacional como un ejemplo para proteger la naturaleza y por consiguiente la preservación de la especie humana. De igual manera contribuye a la construcción del conocimiento científico a partir de la implementación de la IEP.

\section{REFERENCIAS}

Cerda, H. (2007). La investigación formativa en el aula. La Pedagogía como investigación. Bogotá, D.C.: Magisterio.

Chávez. (s.f). Arborización. [Proyecto]. Universidad Nacional de Colombia. Recuperado de https://arquitectura.medellin.unal.edu.co/images/ imagenes/pdf/arborizacin.pdf

El Espectador. (2017). Tasa de deforestación en Colombia. Recuperado de https://www.elespectador.com/ noticias/medio-ambiente/se-disparola-tasa-de-deforestacion-en-colombiaarticulo-701754

García, Y., Ramos, J. y Becerra, J. (2011). Semillas forestales nativas para la restauración ecológica. Biodiversitas, 94(1).12-15.

IDEAM. (2017). Ecosistemas. Recuperado de http://documentacion.ideam.gov.co/ openbiblio/bvirtual/000001/cap7.pdf

IAVH. (2003). Programa de Investigación de Política y Legislación. Sistema de Indicadores de seguimiento de la política de Biodiversidad. Recuperado de http://www.bvsde.paho.org/bvsacd/ cd20/sib.pdf

Instituto Geográfico Agustín CodazziIAGG. (2012). Atlas de la distribución de la propiedad rural en Colombia. Recuperado de https://es.scribd.com/ document/347949611/2012-IGACAtlas-de-La-Distribucion-de-LaPropiedad-Rural-en-Colombia 
Navarro, L. (2016). El mejoramiento integral en el campo de los tratamientos urbanísticos de barrios con problemáticas de crecimiento informal y riesgos ambientales. Caso: Sector arroyo El Salao II, Barranquilla-Colombia. Módulo Arquitectura CUC, 17(1). 115-132. http://revistascientificas.cuc.edu.co/index.php/moduloarquitecturacuc/article/view/1531

Renovables verdes. (2017). Consecuencias de la deforestación. Recuperado de https://www.renovablesverdes.com/ deforestacion/

República de Colombia. Ministerio de Ambiente, (2017). Estrategia integral de control a la deforestación y gestión de los bosques (EICDBG). Minambiente. Recuperado de http://www.minambiente.gov.co/images/EICDGB_1.0_ AGOSTO_9_2017.pdf

Reyes, A. y Gutierrez, J. (2010). Los servicios ambientales de la arborización urbana: Retos y aportes para la sustentabilidad de la Ciudad de Toluca. Quivera, 12(1). 96-102. Recuperado de http://www. redalyc.org/articulo.oa?id=40113202009

Rodríguez, L. (2011). Análisis de las condiciones que impiden la plantación de árboles según los prinicipios de la silvicultura urbana en el nororiente de Barranquilla. Módulo Arquitectura CUC, 10(1), 55-83. Recuperado de https://revistascientificas.cuc.edu.co/moduloarquitecturacuc/article/view/130
Sauvé, L. (1999). La educación ambiental entre la modernidad y la posmodernidad: En busca de un marco educativo de referencia integrador. Tópicos. 1(2). 7-27. Recuperado de http://www.ecominga.uqam. ca/PDF/BIBLIOGRAPHIE/GUIDE_ LECTURE_1/5/2.Sauve.pdf

Vargas, W. (2012). Los bosques secos del Valle del Cauca, Colombia: una aproximación a su flora actual. Biota Colombiana. Especial bosques seco en Colombia, 13(2). 102-164. Recuperado de http://www.humboldt. org.co/images/Atlas\%20 de\%20 paramos/Biota13(2)-Bosque_Seco. pdf

Vining, J. y Ebreo, A. (1992). Predicting Recycling Behavior from Global and Specific Environmental Attitudes and Changes in Recycling Opportunities. Journal of Applied Social Psychology, 22(20), 1580-1607.

WWF. (2017). 15 especies colombianas de árboles amenazados. Recuperado de http://www.wwf.org. co/?uNewsID=299073

WWF. (2018). Principales causas de la deforestación. Recuperado de https://sostenibilidad.semana. com/medio-ambiente/multimedia/ principales-causas-deforestacion-mundo-segun-wwf/32971 
Fig. 4. Palmas en peligro.

Palmas de moriche.

Fuente: (c) Fernando Trujillo WWF (2017)

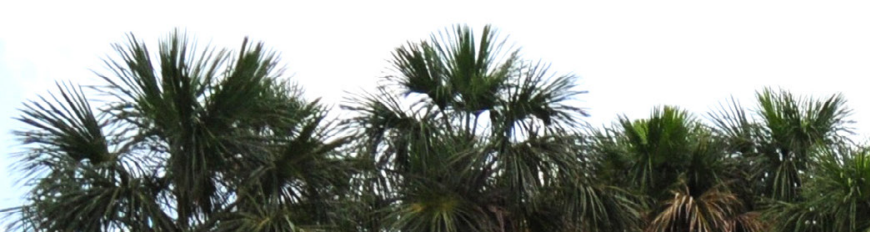

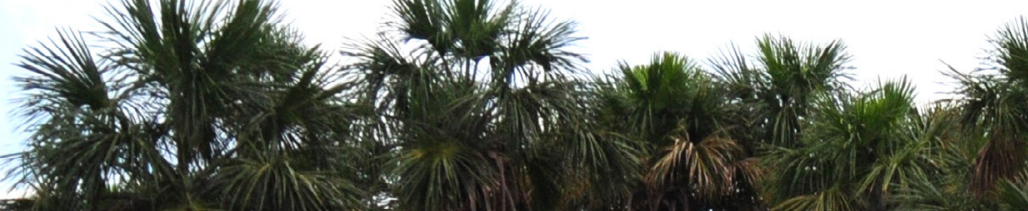

